\title{
Systemic Approaches to Modifying Quinolinic Acid Striatal Lesions in Rats
}

\author{
M. Flint Beal, Neil W. Kowall, Kenton J. Swartz, Robert J. Ferrante, and Joseph B. Martin \\ Neurology Service, Massachusetts General Hospital, and Department of Neurology, Harvard Medical School, Boston, \\ Massachusetts 02114
}

\begin{abstract}
Quinolinic acid (QA) is an endogenous excitotoxin present in mammalian brain that reproduces many of the histologic and neurochemical features of Huntington's disease (HD). In the present study we have examined the ability of a variety of systemically administered compounds to modify striatal QA neurotoxicity. Lesions were assessed by measurements of the intrinsic striatal neurotransmitters substance $P$, somatostatin, neuropeptide $Y$, and GABA. Histologic examination was performed with Nissl stains. The antioxidants ascorbic acid, beta-carotene, and alpha-tocopherol administered s.c. for $3 \mathrm{~d}$ prior to strlatal QA lesions had no significant effect. Other drugs were administered i.p. $1 / 2 \mathrm{hr}$ prior to QA striatal lesions. The following were ineffective in blocking QA excitotoxicity: allopurinol, 50 and $100 \mathrm{mg} / \mathrm{kg}$; ketamine, $75 \mathrm{mg} / \mathrm{kg}$; nimodipine, 2,4, and $10 \mathrm{mg} / \mathrm{kg}$; baclofen, $10 \mathrm{mg} / \mathrm{kg}$; 2-amino-5-phosphonovalerate, $50 \mathrm{mg} / \mathrm{kg}$; and 2-amino-7-phosphonoheptanoate, $50 \mathrm{mg} / \mathrm{kg}$. Oral taurine administration for 4 weeks resulted in significantly increased levels of brain taurine but had no significant effect in blocking QA neurotoxicity. Systemic administration of the noncompetitive $\mathrm{N}$-methyl-D-aspartate (NMDA) antagonist MK-801 resulted in a dose-responsive protection against $Q A$ toxicity, with complete block at a dose of $4 \mathrm{mg} / \mathrm{kg}$. If the pathogenesis of $H D$ involves QA or another excitotoxin acting at the NMDA receptor, it is possible that MK-801 could retard the degenerative process.
\end{abstract}

Huntington's disease (HD) is an autosomal dominant degenerative neurologic disorder in which there is progressive neuronal depletion, particularly in the basal ganglia. There is no available effective treatment to retard or halt the degenerative process. One hypothesis of the pathogenesis of HD is that an excitotoxin may be involved (Schwarcz et al., 1984). This hypothesis was advanced following the finding that kainic acid striatal lesions mimic many of the neurochemical and histopathologic features of HD (Coyle and Schwarcz, 1976; McGeer and McGeer, 1976). Kainic acid, however, is not endogenously present in mammalian brain and does not spare somatostatinneuropeptide Y neurons, which are spared in HD (Beal et al., 1985). Schwarcz and colleagues (1983) found that quinolinic acid (QA) an endogenous metabolite of tryptophan present in

\footnotetext{
Received Oct. 9, 1987; revised Feb. 10, 1988; accepted Mar. 22, 1988.

This work was supported by NINCDS Grant 16367 and the Julieanne Dorn Fund. K. J. S. is an M.D., Ph.D. student at Boston University.

Correspondence should be addressed to Dr. M. Flint Beal, Neurology Research 4, Massachusetts General Hospital, Boston, MA 02114.

Copyright (C) 1988 Society for Neuroscience $0270-6474 / 88 / 103901-08 \$ 02.00 / 0$
}

mammalian brain, is an excitotoxin which produces axon-sparing striatal lesions. We found that this compound produced a more exact model of HD than kainic acid, since the lesions were accompanied by a relative sparing of somatostatin-neuropeptide Y neurons (Beal et al., 1986a).

If an excitotoxin is involved in the pathogenesis of HD, then agents that modify excitotoxin lesions in vivo could potentially be efficacious as therapeutic agents in HD. The best form of therapy from a practical standpoint would be a drug that could be administered systemically, preferably by an oral route. In the present study we have therefore examined the ability of a variety of systemically administered drugs to modify QA striatal neurotoxicity.

\section{Materials and Methods}

Animals. Male Sprague-Dawley rats (Charles River) weighing 150-170 gm were housed in individual cages with ad libitum access to chow and water. A 12:12 hr light-dark cycle was maintained (lights on $0600 \mathrm{hr}$ ), and temperature and humidity were controlled.

Chemicals. QA, allopurinol, taurine, ascorbic acid, beta-carotene, and alpha-tocopherol were purchased from Sigma (St. Louis, MO). Nimodipine was provided by Miles Pharmaceuticals (West Haven, CT). Baclofen was donated by CIBA-GEIGY Corp (Summit, NJ). MK-801 was generously provided by Dr. L. L. Iversen of Merck Sharpe and Dohme. 2-Amino-7-phosphonoheptanoic acid (APH) and 2-amino-5-phosphonovaleric acid (APV) were purchased from Cambridge Biochemicals. Ketamine was purchased from Parke-Davis (Morris Plains, NJ).

Lesion technique. Rats were pretreated with various doses of drugs i.p. $1 / 2 \mathrm{hr}$ before lesions were made. Eight animals were examined in each group unless otherwise specified. Baclofen, APV, APH, and allopurinol were dissolved in normal saline. The highest doses of baclofen $(30 \mathrm{mg} / \mathrm{kg})$ and allopurinol $(250 \mathrm{mg} / \mathrm{kg})$ required dissolving the drugs in $1 \mathrm{~N}$ sodium hydroxide. Controls for these experiments received an equal amount of vehicle. Nimodipine was dissolved in 33\% ethanol/ normal saline, and ketamine was supplied in normal salinc with 0.1 $\mathrm{mg} / \mathrm{ml}$ benzethonium chloride as a preservative. Beta-carotene and alpha-tocopherol were dissolved in olive oil, and ascorbic acid was administered in $\mathrm{H}_{2} \mathrm{O}$ s.c. for $3 \mathrm{~d}$ before lesions according to the protocol of Perry et al. (1985). Taurine was administered orally to rats by administration of the amino acid $(0.9 \%$ final concentration, $0.07 \mathrm{M})$ to the drinking water according to the protocol of Sanberg et al. (1979). Rats received taurine or tap water for 4 weeks prior to lesions and 1 week after lesions. One group of taurine-treated rats and controls received no lesions in order to determine whether taurine treatment itself had any effect on any of the neurochemical parameters examined. Ten animals were examined in each group. Ten to $15 \mathrm{~min}$ before lesions were made rats were anesthetized with pentobarbital, $50 \mathrm{mg} / \mathrm{kg}$, i.p. Quinolinic acid was dissolved in $0.1 \mathrm{M}$ phosphate and neutralized to $\mathrm{pH} 7.4$ with $0.1 \mathrm{M} \mathrm{NaOH}$. Injections were made with a $10 \mu \mathrm{l}$ Hamilton syringe fitted with a 30 gauge blunt-tipped needle into the left striatum at the coordinates $8.4 \mathrm{~mm}$ anterior, $2.6 \mathrm{~mm}$ lateral, and $4.5 \mathrm{~mm}$ ventral for the dura (Beal et al., 1986a). QA, $240 \mathrm{nmol}$, was injected in $1 \mu$ l over 2 min, the needle left in place for an additional $2 \mathrm{~min}$, and then slowly withdrawn. One week after the injection, the animals were sacrificed, and their brains were removed and sectioned at $2 \mathrm{~mm}$ intervals as 


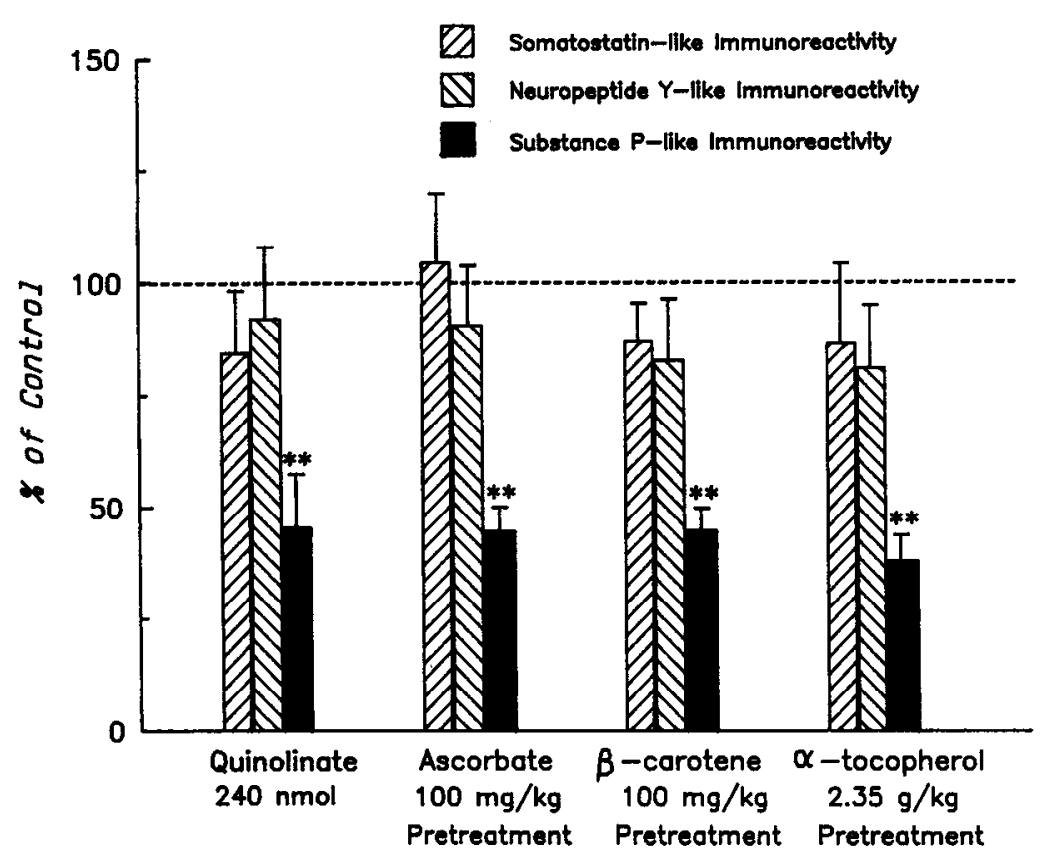

Figure 1. Effects of free-radical blockers on QA striatal lesions. Animals were treated with either saline or ascorbate, beta-carotene, or alpha-tocopherol s.c. for $3 \mathrm{~d}$ prior to QA lesions $(n=8)$. Substance P-like immunoreactivity was significantly reduced in all cases, and there was no beneficial effect $\left({ }^{* *} p<\right.$ 0.01 ).

described previously (Beal et al., 1986a). Both striata were dissected and placed in chilled $0.1 \mathrm{M} \mathrm{HCl}$. Brains for histology were placed in $4 \%$ buffered paraformaldehyde for $24 \mathrm{hr}$ and then transferred to $20 \%$ glycerol, $2 \%$ dimethylsulfoxide in $0.1 \mathrm{M}$ phosphate, $\mathrm{pH} 7.3$, as a cryoprotectant. These brains were subsequently sectioned at $50 \mu \mathrm{m}$ intervals and stained with cresyl violet.

Neurochemical assays. Striatal samples were boiled for $10 \mathrm{~min}$ and centrifuged, and aliquots of supernatant were lyophilized. Extracts were reconstituted in neutral buffer and assayed for somatostatin-like immunoreactivity (SLI), neuropeptide Y-like immunoreactivity (NPYLI), and substance P-like immunoreactivity (SPLI) as previously described (Arnold et al., 1982; Beal et al., 1986b; Beal and Mazurek, 1987). Samples for amino acid analysis were reconstituted in $0.065 \mathrm{M}$ borate buffer, pH 9.0, and assayed by high-performance liquid chromatography with electrochemical detection as recently described (Ellison et al., 1987). For rapid analysis of GABA, the pH was adjusted to 6.0 with a $46 \%$ methanol, $0.1 \mathrm{M}$ phosphate buffer, allowing a total run time of $12 \mathrm{~min}$.
Under these conditions, ethanolamine migrates after GABA. Proteins were measured on the pellets using a fluorimetric assay.

Statistics. All results are expressed as means \pm SEM. Comparisons were made among groups by 1 -way analysis of variance.

\section{Results}

Initial experiments were carried out to assess whether a variety of free-radical blockers could ameliorate QA striatal neurotoxicity. Pretreatment with ascorbate $(100 \mathrm{mg} / \mathrm{kg})$, beta-carotene $(100 \mathrm{mg} / \mathrm{kg})$, and alpha-tocopherol $(2.35 \mathrm{gm} / \mathrm{kg})$ was carried out for $3 \mathrm{~d}$ with s.c. injections, while controls received normal saline. SPLI was measured to quantitate the amount of striatal damage. As depicted in Figure 1, pretreatment with the free-radical blockers had no significant effect in ameliorating QA neurotox-
Figure 2. Effects of allopurinol and ketamine on QA striatal lesions. Animals were given saline or the drugs i.p. 30 min prior to QA injections $(n=8)$. Both SPLI and GABA were significantly reduced in each group with no amelioration of toxicity $\left.{ }^{* *} p<0.01\right)$.

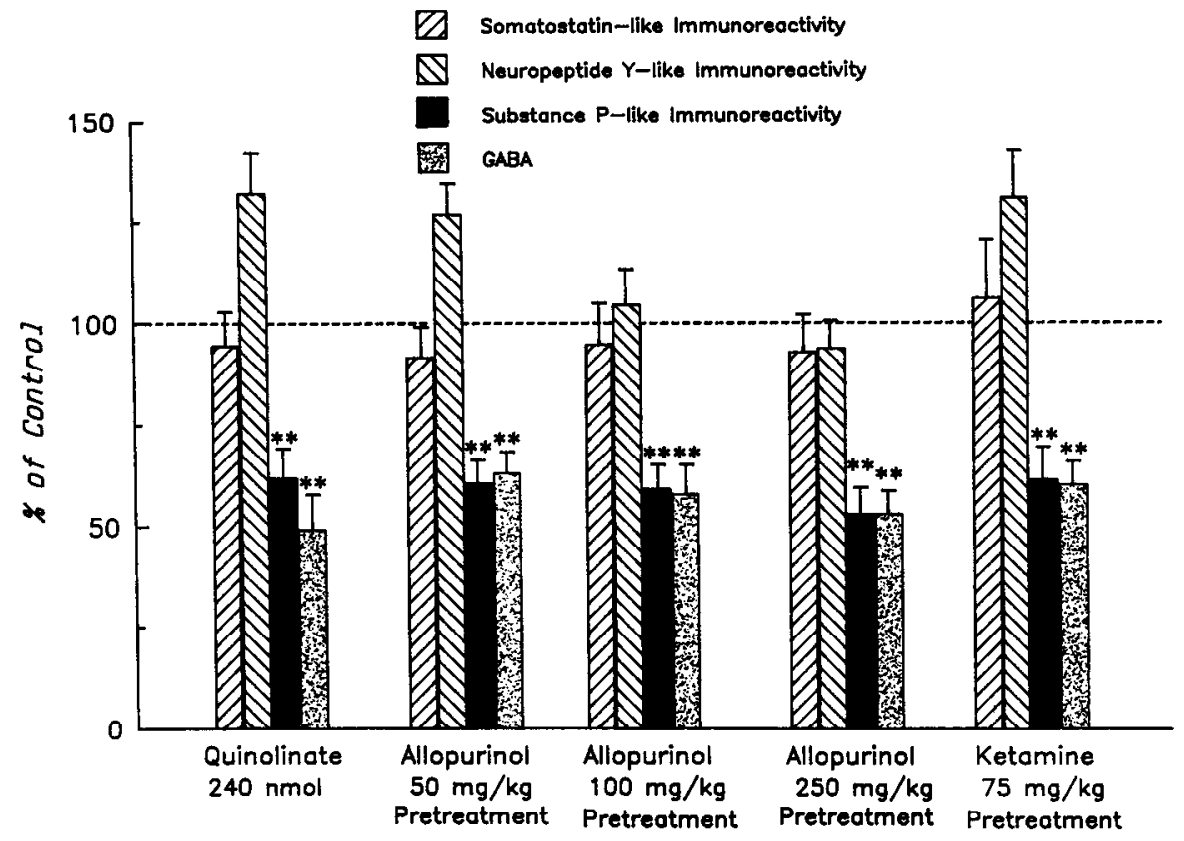




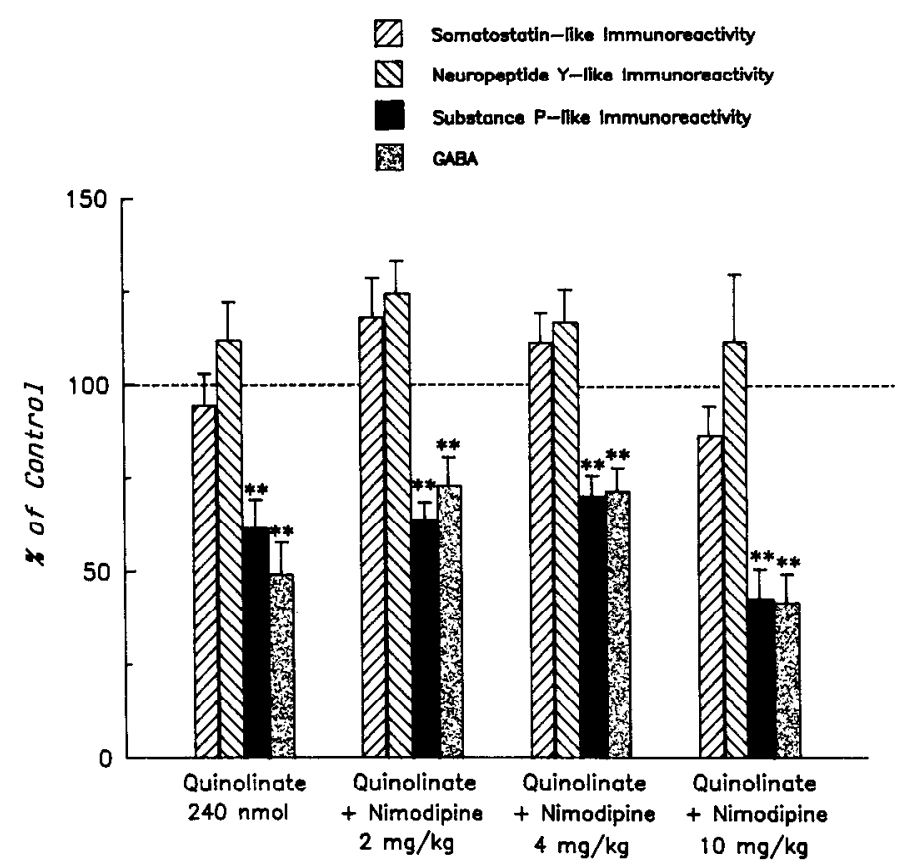

Figure 3. Effects of nimodipine on QA striatal lesions. Rats were treated with saline or varying doses of nimodipine $30 \mathrm{~min}$ prior to QA lesions $(n=8)$. Both SPLI and GABA were significantly reduced in all groups, and there was no beneficial effect $(* * p<0.01)$.

icity. In a second experiment, the effects of i.p. allopurinol and ketamine pretreatment $1 / 2 \mathrm{hr}$ prior to lesions was examined (Fig. 2 ). These drugs were administered in either the maximal dose that could be solubilized for systemic administration (allopurinol) or at the LD50 dose (ketamine) (Marietta et al., 1977). Both GABA and SPLI were examined as independent measures of the extent of striatal lesions. Neither allopurinol nor ketamine had any significant effect at the maximally tolerated doses. In a third experiment, the effects of i.p. pretreatment with the calcium channel blocker nimodipine were compared with saline controls. Nimodipine at doses of 2,4 , and $10 \mathrm{mg} / \mathrm{kg}$ had no significant effect (Fig. 3).

Pretreatment of rats with oral taurine resulted in significant increases in striatal taurine levels from $115 \pm 2.1$ to $158 \pm 5.6$ $\mathrm{nmol} / \mathrm{mg}$ protein $(p<0.01)$. This pretreatment, however, had no significant effect on any of the other neurochemical parameters examined (Fig. 4). Pretreatment with taurine had no significant effect in blocking reductions of GABA and SPLI induced by QA lesions. Although striatal taurine concentrations were increased in animals receiving pretreatment, the percentage taurine depletion following QA lesions was comparable in animals receiving either pretreatment with taurine or tap water $(27.8 \pm 6.2$ vs $29.6 \pm 5.1 \%)$.

I he effects of pretreatment with i.p. baclofen, APV, and APH were compared with saline controls. Baclofen was administered at the maximally tolerated dose $(30 \mathrm{mg} / \mathrm{kg})$, which was at the LD50, and twice levels reported to show toxic behavioral effects (Ulloque et al., 1986). As shown in Figure 4, systemic administration of these compounds had no significant effect on QA neurotoxicity. In contrast to systemic APV administration, coinjection of an equimolar amount of APV in the striatum completely blocked QA toxicity (Fig. 5). The effects of systemic pretreatment with the NMDA antagonist MK-801 are shown in Figure 6. There was no protective effect at $1 \mathrm{mg} / \mathrm{kg}$, but there was a dose-dependent protection with increasing doses. Total protection was achieved with a dose of $4 \mathrm{mg} / \mathrm{kg}$. This effect was verified by histologic examination of the lesions with cresyl violet-stained sections (Fig. 7). These showed a dose-related block of histopathologic effects such that QA neurotoxicity was completely blocked at a dose of $4 \mathrm{mg} / \mathrm{kg}$.

\section{Discussion}

The possibility that excitotoxins may play a role in the pathogenesis of a variety of human neurologic illnesses has been strengthened by recent findings. The most convincing data has shown that ischemia, hypoglycemic, and epileptic neuropathologic findings can be blocked with excitatory amino acid antagonists (Schwarcz and Meldrum, 1985). HD was among the

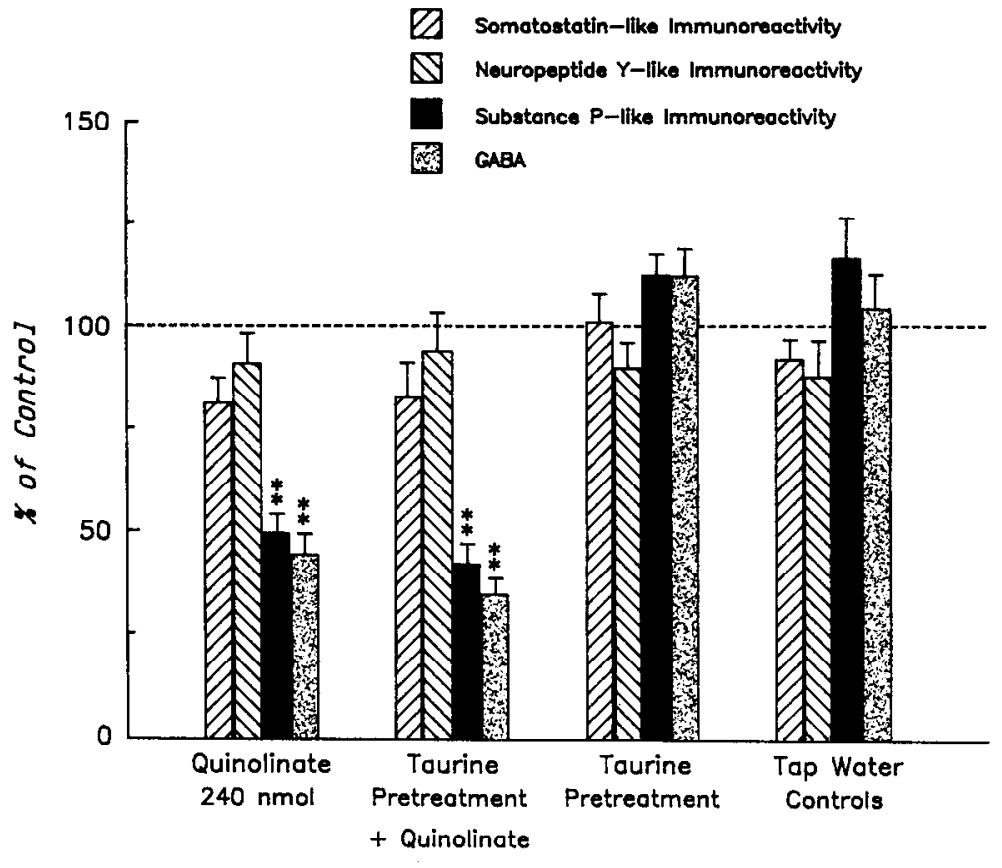

Figure 4. Effects of pretreatment with taurine. Taurine $(0.07 \mathrm{M})$ was administered in drinking water for 4 weeks before and 1 week after QA lesions ( $n$ $=10$ ). Taurine treatment itself did not alter the neurochemical parameters examined as compared with controls (groups 3 and 4) except for a significant increase in striatal taurine content (see Results). Compared with controls there was no significant protective effect against QA-induced GABA and SPLI depletions (groups 1 and 2$)\left({ }^{* *} p<0.01\right.$ ). 
Figure 5. Effects of pretreatment with baclofen, 2-amino-5-phosphonovalerate (APV) and 2-amino-7-phosphonoheptanoate (APH) on QA striatal lesions. Animals received saline or drugs i.p. 30 min prior to QA striatal lesions $(n=8)$. Both SPLI and GABA were significantly reduced by the lesions, but there was no beneficial effect of any of the pretreatments $\left({ }^{* *} p<0.01\right)$; however, co-injection of equimolar APV with QA completely blocked striatal toxicity.

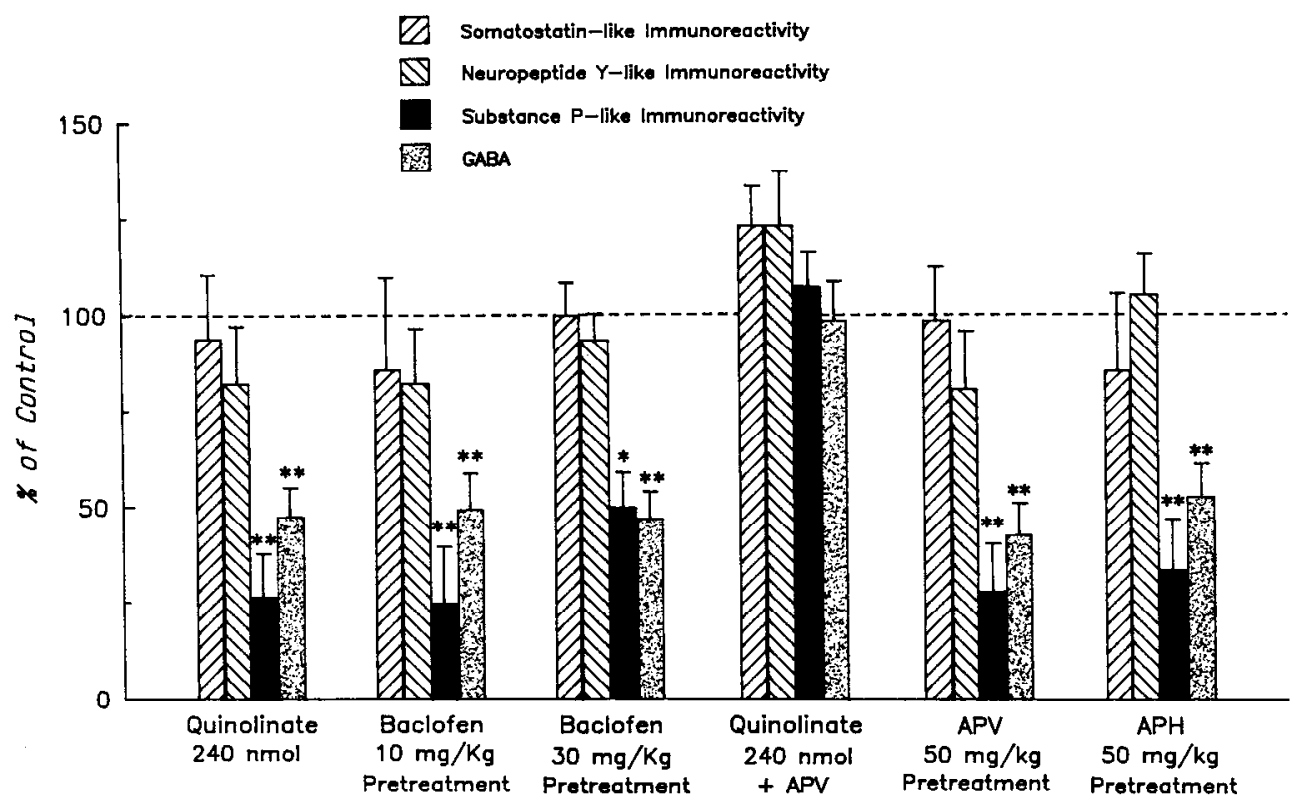

first neurologic illnesses in which a possible excitotoxic etiology was proposed (Coyle and Schwarcz, 1976; McGeer and McGeer, 1976). QA is one of the most promising candidates as an endogenous excitotoxin (Schwarcz et al., 1984). Previous work has shown that QA striatal lesions reproduce many of the neurochemical and histologic features of HD (Schwarcz et al., 1983; Beal et al., 1986a). If an excitotoxin is involved in the pathogenesis of HD, then agents that block its toxicity might be efficacious in retarding the degenerative process.

The exact cellular mechanisms of excitotoxin-mediated neuronal toxicity are as yet unknown. Partially reduced and thereby activated oxygen species have heen implicated as mediators of cell injury in a variety of pathologic circumstances (Hill and Burke, 1984; McCord, 1985; Halliwell and Gutteridge, 1986). $N$-Methyl-4-phenyl-1,2,3,6-tetrahydropyridine (MPTP) toxicity can be partially blocked by pretreatment with antioxidants (Perry et al., 1985). Kainate toxicity to cerebellar neurons in culture can be prevented by inhibiting the enzyme xanthine oxidase, a cellular source of superoxide radicals, as well as with other agents that detoxify free radicals (Dykens et al., 1987). We therefore examined the effects of a variety of antioxidants, including beta-carotene, ascorbic acid, and alpha-tocopherol on QA striatal toxicity. None of these agents had any therapeutic effect at the doses administered. We also examined the effects of allopurinol since this compound has protective effects in models of ischemic damage and against kainate toxicity in vitro (DeWall et al., 1971; McCord, 1985; Dykens et al., 1987). It has bcen shown that xanthine dehydrogenase is converted to xanthine oxidase in vivo in ischemic tissues and that xanthine oxidase then generates free radicals (McCord, 1985; Engerson et al., 1987). Allopurinol is protective in these circumstances. Allopurinol penetrates into the CNS with levels approximately $50 \%$ of systemic levels (Elion et al., 1966). Despite using allopurinol at much higher doses than those that are effective in vivo in ischemic models $(50 \mathrm{mg} / \mathrm{kg})$, no protective effect was observed (DeWall et al., 1971). These results do not rule out a
Figure 6. Effects of pretreatment with MK-801 prior to QA striatal lesions. MK-801 was administered i.p. $30 \mathrm{~min}$ before striatal QA lesions $(n=8)$. MK801 at a dose of $1 \mathrm{mg} / \mathrm{kg}$ had no effect, but a dose-dependent blockade of QA toxicity was seen with increasing doses with complete protection at a dose of 4 $\mathrm{mg} / \mathrm{kg}\left({ }^{*} p<0.05,{ }^{* *} p<0.01\right)$.

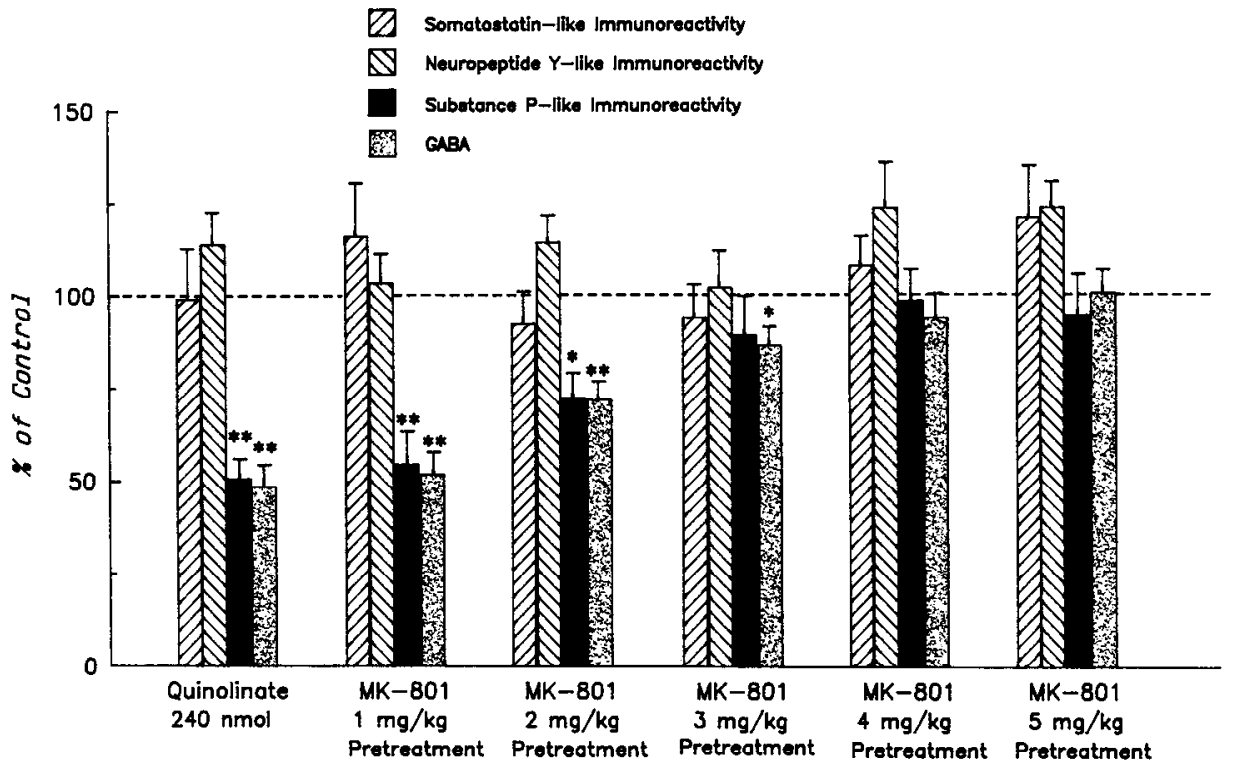



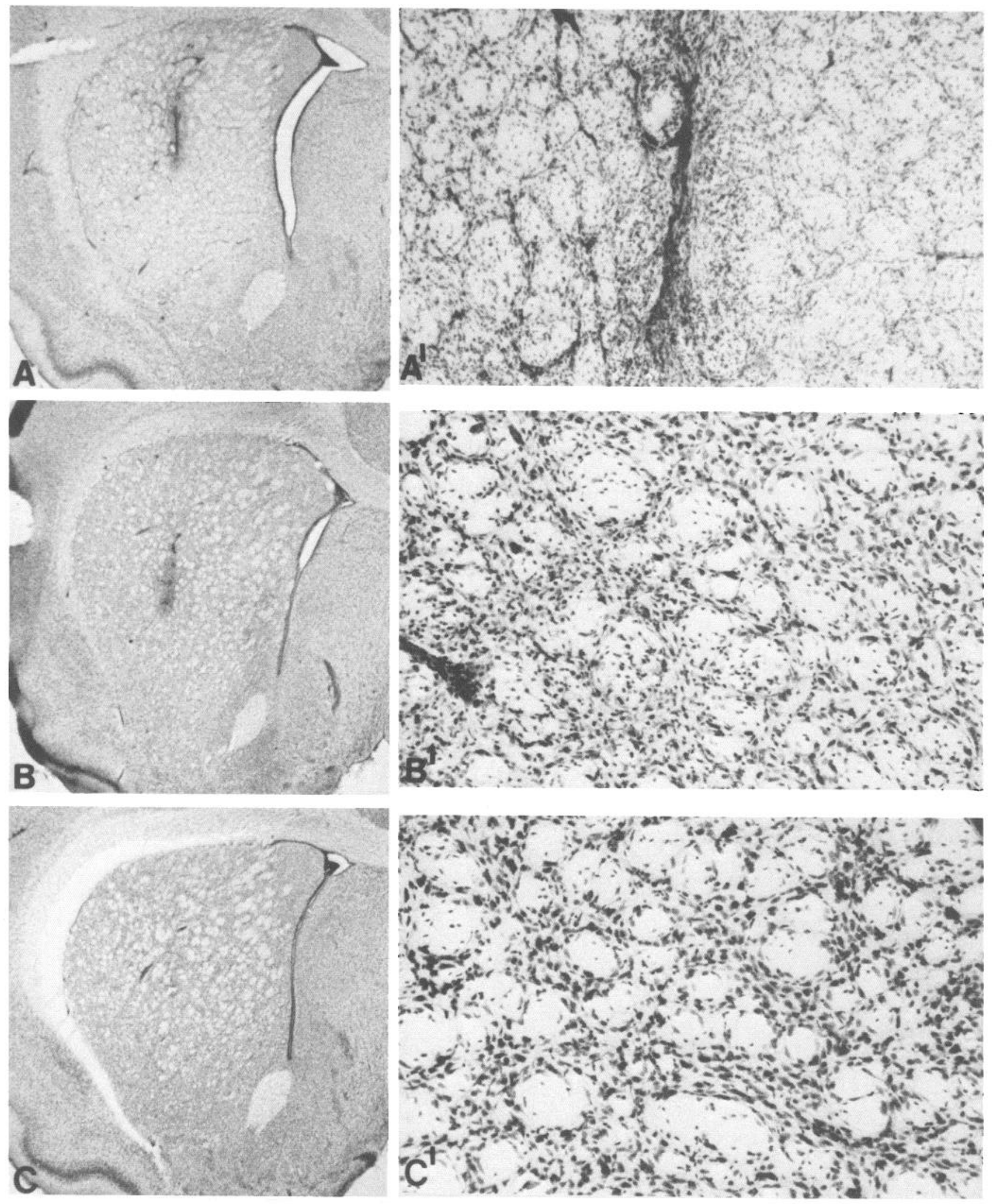

Figure 7. Histological effects of systemic MK-801 pretreatment on QA toxicity. A, A zone of total neuronal depletion surrounds the injection site (asterisk) in the striatum after an injection of $240 \mathrm{nmol}$ of QA. $A_{i}$, Higher-power view adjacent to the injection site. $B$, The region of total neuronal depletion is diminished after pretreatment with $3 \mathrm{mg} / \mathrm{kg}$ MK-801, with some neurons visible adjacent to the injection $\left(B_{l}\right)$. $C$, The injection site is difficult to define in rats pretreated with higher doses of MK-801 $(5 \mathrm{mg} / \mathrm{kg})$. Little if any neuronal depletion is evident surrounding the injection site in these cases $\left(C_{l}\right)$. Cresyl violet stain. Original magnification: low-power views, $\times 100$; higher-power views, $\times 400$. 
possible role of free radicals in excitotoxic damage in vivo since higher doses might show an effect; however, it is unlikely that allopurinol will be useful therapeutically since much lower doses $(10-20 \mathrm{mg} / \mathrm{kg})$ are known to be toxic in man.

A lethal alteration in intracellular ion concentrations has recently been investigated as the mechanism of excitotoxic neurond death. Interest has focused on both sodium and chloride, as well as calcium which is known to be important in a variety of types of cell death (Schanne et al., 1979; Rothman, 1985). It has been suggested that continuous neuronal depolarization leads to a depletion of neuronal energy stores and inability to maintain neuronal ionic gradients of sodium and chloride. Consistent with this suggestion, an early morphologic finding accompanying excitotoxic damage is dendritic swelling (Schwarcz et ai., 1984). Experiments using retinal slices and hippocampal neurons in vitro demonstrated that removal of extracellular calcium had no effect on excitotoxicity, while removal of sodium or chloride were protective (Rothman, 1985; Olney et al., 1986b). More recent studies of Choi (1987), as well as others (Garthwaite and Garthwaite, 1986), have shown that there are 2 phases of neurotoxicity. There is an early sodium-dependent toxicity and a later calcium-dependent neurotoxicity. Calcium channels are directly activated by NMDA agonists (McDermott et al., 1986; Kudo et al., 1987; Murphy et al., 1987). We therefore examined the ability of the calcium channel blocker nimodipine to modify QA striatal neurotoxicity. A dose of $1 \mathrm{mg} / \mathrm{kg}$ in gerbils results in brain levels at $1 \mathrm{hr}$ that are 20 -fold greater than those needed to mediate a maximal pharmacologic effect (Heffez et al., 1985). Despite our using doses many fold in excess of this, we observed no beneficial effect. There was a trend towards worsening toxicity at the highest dose, consistent with the results of Price et al. (1985), who found that nimodipine worsened NMDA excitotoxic lesions in the arcuate nucleus. This is consistent with recent work suggesting that the voltage-dependent calcium channels are distinct from the NMDA-linked calcium channels and that only the former are blocked by dihydropyridine calcium channel antagonists (Miller, 1987). Nifedipine has been shown to open some calcium channels in cortical slices, which could account for the slight worsening of neurotoxicity seen with the highest dose of nimodipine (Riveros and Orrego, 1986) (Fig. 3). It has also heen shown that nitrendipine has no effect on glutamate-induced calcium accumulation in isolated hippocampal neurons (Kudo and Ogura, 1986) and that flunarizine is ineffective in blocking NMDA excitotoxicity in cerebellar slices (Lehmann, 1987).

Taurine was also found to be ineffective in preventing QA striatal toxicity. Taurine prevents NMDA-induced reductions in extracellular calcium in vivo (Lehman et al., 1984) and has been reported to have small protective effects against kainic acid neurotoxicity (Sanberg et al., 1979). Protective effects of taurine in vitro against QA damage in the hippocampus have been demonstrated, although there was little effect in vivo (French et al., 1986). The present results are consistent with the work of Lehmann et al. (1987), who found no enhancement of kainic acid ncurotoxicity in vivo following depletion of brain taurine levels. Two in vitro studies have also shown no protective effects of taurine against NMDA toxicity (Olney et al., 1986a; Lehmann, 1987).

Another approach to blocking QA striatal toxicity is to attempt to block corticostriatal glutamate release. QA striatal toxicity is abolished by prior decortectomy, suggesting that QA may act synergistically with glutamate in its excitotoxic mechanism (Schwarcz et al., 1984). Baclofen has been shown to inhibit release of glutamate and aspartate from cortical cell slices and has been reported to have a modest effect in blocking kainic acid neurotoxicity (Potashner, 1979; McGeer et al., 1980). Clinical trials with this compound in HD have therefore been undertaken (Shoulson, 1984). Baclofen (1-4.5 mg/kg, i.v.), however, has no effect on depolarizing responses of cat caudate neurons induced by either cortical or thalamic stimulation, despite blocking hyperpolarizing responses, suggesting that it may not block corticostriatal glutamate release (Wilson and Wilson, 1985). Consistent with this, we found no effect of baclofen on QA striatal toxicity using doses (10 or $30 \mathrm{mg} / \mathrm{kg}$ ) that are $10-$ to 30 -fold higher that that being utilized in clinical studies (Shoulson, 1984), and well above that reported to block glutamate release (Potashner, 1979).

The most promising approach to preventing QA toxicity is to block its effects at the excitatory amino acid receptor. It is known that QA activates the NMDA receptor and that its toxicity can be blocked by co-injection of equimolar amounts of the competitive NMDA antagonists APV and APH (Schwarcz et al., 1984). We have replicated this finding in the present study. Unfortunately, these highly charged compounds do not penetrate the blood-brain barrier well. Studies have shown that brain uptake of ${ }^{3} \mathrm{H}-\mathrm{APH}$ is $0.1 \%$ of the total amount injected (Chapman et al., 1983). Systemic administration of large doses, which are efficacious in blocking seizures (Croucher et al., 1982) had no effect on QA striatal toxicity. Systemic administration of large amounts of CPP (a more potent NMDA antagonist), however, are partially effective in blocking striatal QA toxicity (Foster et al., 1987b).

Recent work has shown that several drugs are noncompetitive antagonists of NMDA responses (Anis et al., 1983; Olney et al., 1986a). These drugs appear to bind to a site linked to the ion channel activated by the NMDA receptor, a site considered to be distinct from that which binds NMDA directly (Kemp et al., 1987). The noncompetitive NMDA antagonists include phencyclidine, ketamine, and sigma-opiate benzomorphans. Ketamine has been shown to block $N$-methyl-D,L-aspartate toxicity in the chick embryo retina in vitro (Olney et al., 1986b). In the present experiments, however, there was no significant protective effect at a dose of $75 \mathrm{mg} / \mathrm{kg}$, which was at the LD50. Lees (1987) also found only partial effects of systemic ketamine in blocking OA toxicity in the hippocampus and no effect of local injections. The lack of efficacy of ketamine in vivo probably relates to its low potency, since it is 50 -fold less potent than MK-801 in vitro (Olney et al., 1987).

MK-801 is a noncompetitive NMDA antagonist that was initially discovered while screening compounds for anticonvulsant activity (Wong et al., 1986). In the present studies, it was the only systematically administered compound that was efficacious in blocking quinolinate striatal toxicity. There was no effect at a dose of $1 \mathrm{mg} / \mathrm{kg}$; however, a dose-responsive blockade of excitotoxicity was seen with increasing doses. Complete neurochemical and histologic protection was achieved with a dose of $4 \mathrm{mg} / \mathrm{kg}$. These findings are consistent with the recent report of Foster et al. (1987a, b) that MK-801 can block NMDAinduced neuronal degeneration in both the striatum and hippocampus and QA neurotoxicity in rat striatum (Woodruff et al., 1987). Interestingly MK-801 is effective even if administered 1 or 2 hr after QA lesions (Foster et al., 1987b). MK-801 has 
been shown to block NMDA-mediated calcium influx, and its effects are voltage dependent, consistent with a locus of action at the level of the ionophore (Murphy et al., 1987).

These findings confirm that QA neurotoxicity in vivo is a specific receptor-mediated phenomenon. QA is a better model of HD than kainic acid since it results in relative sparing of somatostatin-neuropeptide $Y$ neurons, which are also spared in HD (Beal et al., 1986a). It must be emphasized, however, that sparing is relative and not absolute since these neurons are killed within the lesion core (Davies and Roberts, 1987). With 1 year lesions, the lesion core is resorbed, and an increased density of somatostatin-neuropeptide $Y$ neurons can be clearly demonstrated (Beal et al., unpublished observations). We have recently found that other NMDA agonists such as L-homocysteate and $N$-methyl-D,L-aspartate can produce similar effects at the appropriate dosage (Beal et al., unpublished observations), consistent with work in cortical cell cultures (Koh et al., 1986). Agents acting at the NMDA receptor could therefore be involved in the pathogenesis of HD. MK- 801 is well absorbed systemically and readily penetrates the blood-brain barrier. A potential disadvantage is that blockade of NMDA receptors may result in psychotomimetic effects (Koek et al., 1987). However, it is possible that in HD there is only a mild increase in NMDA receptor activation since it is a slowly evolving process. If this were the case, it might be possible to attempt to retard the degenerative process with low doses of MK-801 while avoiding psychotomimetic effects.

\section{References}

Anis, N. A., S. C. Berry, N. R. Burton, and D. Lodge (1983) The dissociative anaesthestics, ketamine and phencyclidine, selectively reduce excitation of central mammalian neurones by $\mathrm{N}$-methyl-aspartate. Br. J. Pharmacol. 79: 565-575.

Arnold, M. A., S. M. Reppert, O. Rorstad, S. M. Sagar, H. T. Keutmann, M. J. Perlow, and J. B. Martin (1982) Temporal patterns of somatostatin immunoreactivity in the cerebrospinal fluid of rhesus monkeys: Effect of environmental lighting. J. Neurosci. 2: 674-680.

Beal, M. F., and M. F. Mazurek (1987) Substance P-like immunoreactivity is reduced in Alzheimer's disease cerebral cortex. Neurology 37: 1205-1209.

Beal, M. F., P. E. Marshall, G. D. Burd, D. M. D. Landis, and J. B. Martin (1985) Excitotoxin lesions do not mimic the alteration of somatostatin in Huntington's disease. Brain Res. 361: 135-145.

Beal, M. F., N. W. Kowall, D. W. Ellison, M. F. Mazurek, K. J. Swartz, and J. B. Martin (1986a) Replication of the neurochemical characteristics of Huntington's disease by quinolinic acid. Nature 321: 168-171.

Beal, M. F., M. F. Mazurek, L. J. Lorenz, G. K. Chattha, D. W. Ellison, and J. B. Martin (1986b) An examination of neuropeptide $Y$ postmortem stability in an animal model simulating human autopsy conditions. Neurosci. Lett. 64: 69-74.

Chapman, A. G., J. F. Collins, B. S. Meldrum, and E. Esterberg (1983) Uptake of a novel anticonvulsant compound, 2-amino-7-phosphono$\left[4,5-{ }^{3} \mathrm{H}\right]$ heptanoic acid, into mouse brain. Neurosci. Lett. 37: 75-80.

Choi, D. W. (1987) Ionic dependence of glutamate neurotoxicity. J. Neurosci. 7: 369-379.

Coyle, J. T., and R. Schwarcz (1976) Lesions of striatal neurons with kainic acid provides a model for Huntington's chorea. Nature 263: 244-246.

Croucher, M. J., J. F. Collins, and B. S. Meldrum (1982) Anticonvulsant action of excitatory amino acid antagonists. Science 216:899901.

Davies, S. W., and P. J. Roberts (1987) No evidence for preservation of somatostatin containing neurons after intrastriatal injections of quinolinic acid. Nature 327: 326-329.

DeWall, R. A., K. A. Vasko, E. L. Stanley, and P. Kezdi (1971) Re- sponses of the ischemic myocardium to allopurinol. Am. Heart J. 82: 362-370.

Dykens, J. A., A. Stern, and E. Trenkner (1987) Mechanism of kainate toxicity to cerebellar neurons in vitro is analogous to reperfusion tissue injury. J. Neurochem. 49: 1222-1228.

Elion, G. B., A. Kovensky, G. H. Hitchings, E. Metz, and R. W. Rundles (1966) Metabolic studies of allopurinol, an inhibitor of xanthine oxidase. Biochem. Pharmacol. 15: 863-880.

Ellison, D. W., M. F. Beal, and J. B. Martin (1987) Amino acid neurotransmitters in postmortem human brain analyzed by high performance liquid chromatography with electrochemical detection. J. Neurosci. Methods 19: 305-315.

Engerson, T. D., T. G. McKelvey, D. B. Rhyne, E. B. Boggio, S. J. Snyder, and H. D. Jones (1987) Conversion of xanthine dehydrogenase to oxidase in ischemic rat tissues. J. Clin. Invest. 79: 15641570 .

Foster, A. C., and E. H. F. Wong (1987) The novel anticonvulsant MK-801 binds to the activated state of the N-methyl-D-aspartate receptor in rat brain. Br. J. Pharmacol. 91: 403-409.

Foster, A. C., R. Gill, J. A. Kemp, and G. N. Woodruff (1987a) Systemic administration of MK-801 prevents N-methyl-D-aspartate induced neuronal degeneration in rat brain. Neurosci. Lett. 76: 307311.

Foster, A. C., R. Gill, and G. N. Woodruff (1987b) A delayed excitotoxic mechanism mediates the degeneration of rat striatal neurones caused by N-methyl-D-aspartate and quinolinate. Soc. Neurosci. Abstr. 13: 1030 .

French, E. D., A. Vezzani, W. O. Whetsell, Jr., and R. Schwarcz (1986) Antiexcitotoxic actions of taurine in the rat hippocampus studied in vivo and in vitro. In Excitatory Amino Acids and Epilepsy, R. Schwarcz and Y. Ben-Ari, eds., pp. 349-362, Plenum, New York.

Garthwaite, G., and J. Garthwaite (1986) Neurotoxicity of excitatory amino acid receptor agonists in rat cerebellar slices: Dependence on calcium concentration. Neurosci. Lett. 66: 193-198.

Halliwell, B., and J. M. C. Gutteridge (1986) Oxygen free radicals and iron in relation to biology and medicine: Some problems and concepts. Arch. Biochem. Biophys. 246: 501-514.

Heffez, D. S., T. S. Nowak, and J. V. Passoneau (1985) Nimodipine levels in gerbil brain following parenteral drug administration. J. Neurosurg. 63: 589-592.

Hill, K. E., and R. F. Burke (1984) Influence of vitamin E and selenium on glutathione-dependent protection against microsomal lipid peroxidation. Biochem. Pharmacol. 33: 1065-1068.

Kemp, J. A., A. C. Foster, and E. H. F. Wong (1987) Non-competitive antagonists of excitatory amino acid receptors. TINS 10: 294-298.

Koek, W., J. H. Woods, and P. Ornstein (1987) A simple and rapid method for assessing similarities among directly observable behavioral effects of drugs: PCP-like effects of 2-amino-5-phosphonovalerate in rats. Psychopharmacology 91: 297-304.

Koh, J., S. Peters, and D. W. Choi (1986) Neurons containing NADPHdiaphorase are selectively resistant to quinolinate toxicity. Science 234: 73-76.

Kudo, Y., and A. Ogura (1986) Glutamate-induced increase in intracellular calcium concentration in isolated hippocampal neurones. $\mathrm{Br}$. J. Pharmacol. 89: 191-198.

Kudo, Y., K. Ito, H. Miyakawa, Y. Izumi, A. Ogura, and H. Kato (1987) Cytoplasmic calcium elevation in hippocampal granule cell induced by perforant path stimulation and L-glutamate application. Brain Res. 407: $168-172$.

Lees, G. J. (1987) Effects of ketamine on the in vivo toxicity of quinolinate and $\mathrm{N}$-methyl-D-aspartate in the rat hippocampus. Neurosci. Lett. 78: 180-186.

Lehmann, A. (1987) Pharmacological protection against the toxicity of N-methyl-D-aspartate in immature rat cerebellar slices. Neuropharmacology 26: 1751-1761.

Lehmann, A., H. Hagberg, and A. Hamberger (1984) A role for taurine in the maintenance of homeostasis in the central nervous system during hyperexcitation? Neurosci. Lett. 52: 341-346.

Lehmann, A., H. Hagberg, R. J. Huxtable, and M. Sandberg (1987) Reduction of brain taurine: Effects on neurotoxic and metabolic actions of kainate. Neurochem. Int. 10:265-274.

Loo, P. A., A. F. Braunwalder, M. Williams, and M. A. Sills (1987) The novel anticonvulsant MK-801 interacts with central phencyclidine recognition sites in rat brain. Eur. J. Pharmacol. 135: 261-263. 
MacDermott, A. B., M. L. Mayer, G. L. Westbrook, S. J. Smith, and J. L. Barker (1986) NMDA-receptor activation increases cytoplasmic calcium concentration in cultured spinal cord neurones. Nature 321: 519-522.

Marietta, M. P., W. L. Way, N. Castagnoli, and A. J. Trevor (1977) On the pharmacology of the ketamine enantiomorphs in the rat. J. Pharmacol. Exp. Ther. 202: 157-165.

McCord, J. M. (1985) Oxygen-derived free radicals in postischemic tissue injury. N. Engl. J. Med. 312: 159-163.

McGeer, E. G., and P. L. McGeer (1976) Duplication of biochemical changes of Huntington's chorca by intrastriatal injections of glutamic and kainic acids. Nature 263: 517-519.

McGeer, E. G., A. Takubovic, and E. A. Singh (1980) Ethanol, baclofen, and kainic acid neurotoxicity. Exp. Neurol. 69: 359-364.

Miller, R. J. (1987) Multiple calcium channels and neuronal function. Science 235: 46-52.

Murphy, S. N., S. A. Thayer, and R. J. Miller (1987) The effects of excitatory amino acids on intracellular calcium in single mouse striatal neurons in vitro. J. Neurosci. 7: 4145-4158.

Olney, J. W., M. T. Price, T. A. Fuller, J. Labruyere, L. Samson, M. Carpenter, and K. Mahan (1986a) The anti-excitotoxic effects of certain anesthetics, analgesics and sedative-hypnotics. Neurosci. Lett. 68: 29-34.

Olney, J. W., M. T. Price, L. Samson, and J. Labruyere (1986b) The role of specific ions in glutamate neurotoxicity. Neurosci. Lett. 65: $65-71$.

Olney, J., M. Price, K. S. Salles, J. Labruyere, and G. Frierdich (1987) MK-801 powerfully protects against $\mathrm{N}$-methyl aspartate neurotoxicity. Eur. J. Pharmacol. 41:357-361.

Perry, T. L., V. W. Yong, R. M. Clavier, K. Jones, J. M. Wright, J. G. Foulk, and R. A. Wall (1985) Partial protections from the dopaminergic neurotoxin $\mathrm{N}$-methyl-4-phenyl-1,2,3,6-tetrahydropyridine by four different antixodiants in the mouse. Neurosci. Lett. 60: 109114.

Potashner, S. J. (1979) Baclofen: Effects on amino acid release and metabolism in slices of guinea pig cerebral cortex. J. Neurochem. 32: 103-109.

Price, M. T., J. W. Olney, L. Samson, and J. Labruyere (1985) Calcium influx accompanies but does not causc excitotoxin-induccd ncuronal necrosis in retina. Brain Res. Bull. 14: 369-375.
Riveros, N., and F. Orrego (1986) N-methylaspartate-activated calcium channels in rat brain cortex slices. Effect of calcium channel blockers and of inhibitory and depressant substances. Neuroscience 17: 541-546.

Rothman, S. M. (1985) The neurotoxicity of excitatory amino acid is produced by passive chloride influx. J. Neurosci. 5: 1483-1489.

Sanberg, P. R., W. Staines, and E. G. McGeer (1979) Chronic taurine effects on various neurochemical indices in control and kainic acidlesioned neostriatum. Brain Res. 161: 367-370.

Schanne, F. A. X., A. B. Kane, E. E. Young, and J. L. Farber (1979) Calcium dependence of toxic cell death: A final common pathway. Science 206: 700-702.

Schwarcz, R., and B. Meldrum (1985) Excitatory amino acid antagonists provide a therapeutic approach to neurological disorders. Lancet $2: 140-143$.

Schwarcz, R., W. O. Whetsell, and R. M. Mangano (1983) Quinolinic acid: An endogenous metabolite that produces axon-sparing lesions in rat brain. Science 219: 316-318.

Schwarcz, R., A. C. Foster, E. D. French, W. O. Whetsell, and C. Kohler (1984) Excitotoxin models for neurogenerative disorders. Life Sci. 35: $19-32$

Shoulson, I. (1984) Huntington's disease: Anti-neurotoxic therapeutic strategies. K. Fuxe, P. Roberts, and R. Schwarcz, eds., pp. 343-353, MacMillan, London.

Ulloque, R. A., A. Y. Chweh, and E. A. Swinyard (1986) Effects of gamma-aminobutyric acid (GABA) receptor agonists on the neurotoxicity and anticonvulsant activity of barbiturates in mice. J. Pharmacol. Exp. Ther. 237: 468-472.

Wilson, J. S., and J. A. Wilson (1985) Baclofen attenuates hyperpolarizing but not depolarizing responses of caudate neurons in cat. Brain Res. 342: 396-400.

Wong, E. H. F., J. A. Kemp, T. Priestly, A. R. Knight, G. N. Woodruff, and L. L. Iversen (1987) The anticonvulsant MK-801 is a potent N-methyl-D-aspartate antagonist. Proc. Natl. Acad. Sci. USA 83: 7104-7108

Wondruff, G. N., A. C. Foster, R. Gill, J. A. Kemp, E. H. F. Wong, and L. L. Iversen (1987) The interaction between MK-801 and receptors for $\mathrm{N}$-methyl-D-aspartate: Functional consequences. Neuropharmacology 26(7B): 903-909. 